\title{
Antitumor Activity of DLX1008, an Anti-VEGFA Antibody Fragment with Low Picomolar Affinity, in Human Glioma Models ${ }^{[}$
}

\author{
Emese Szabó, ${ }^{1}$ Douglas J. Phillips, ${ }^{1}$ Miriam Droste, Andrea Marti, Titus Kretzschmar, \\ Abdijapar Shamshiev, and Michael Weller \\ Laboratory of Molecular Neuro-Oncology, Department of Neurology, University Hospital and University of Zurich (E.S., M.W.), \\ and Cell Medica Switzerland AG, Schlieren (D.J.P., M.D., A.M., T.K., A.S.), Zurich, Switzerland
}

Received November 6, 2017; accepted February 20, 2018

\begin{abstract}
Angiogenesis mediated by vascular endothelial growth factor (VEGF) is a hallmark of glioblastoma. Based on the response rate and improved progression-free survival, although not on overall survival, the 149-kDa anti-VEGF-A IgG antibody bevacizumab (Avastin) has been approved in the United States and Japan for recurrent glioblastoma and in Japan for newly diagnosed glioblastoma; however, it is not approved in the EU. Here we characterize the biologic activity of DLX1008, a 26-kDa antiVEGF-A single-chain antibody fragment that shows 30 -fold stronger affinity to human VEGF-A than bevacizumab. The small molecular size of DLX1008 is predicted to result in improved target coverage over bevacizumab. DLX1008 showed
\end{abstract}

superiority to bevacizumab in the inhibition of VEGF-A binding to VEGF receptor (VEGFR) 1 in enzyme-linked immunosorbent assay by a factor of around 10 and comparable efficacy for the inhibition of VEGF-A-stimulated VEGFR2 dimerization. In a tubeformation assay with human cerebral microvascular endothelial cells, DLX1008 was at least as active as bevacizumab. In vivo, DLX1008 delayed growth in a mouse subcutaneous U87 xenograft model $(P=0.0021)$ and improved survival in a mouse orthotopic U87 xenograft model $(P=0.00026)$. Given the exceptionally high affinity and small molecular size of DLX1008, these data warrant further clinical development of DLX1008 as an antiangiogenic agent in glioblastoma.

\section{Introduction}

Intense and aberrant blood vessel development, defined as tumor-associated angiogenesis, is a common feature of gliomas, intrinsic brain tumors thought to be derived from neuroglial progenitor cells. Accordingly, the sensitivity of gliomas to antiangiogenic drugs has been extensively studied, given that the tumor vasculature provides nutrient supply for tumor growth, supports glioma cell invasion, and represents a filter barrier to therapeutic molecules and tumor-infiltrating immune cells (Batchelor et al., 2014).

VEGF is a growth factor that plays a key role in angiogenesis. Along with its stimulatory activity on tumor vessel formation, VEGF may exert tumor-intrinsic survival properties mediated by both VEGF receptors 1 and 2 (Knizetova et al., 2008; von Baumgarten et al., 2011; Lu et al., 2012; Szabo et al., 2016).

Although various VEGF inhibitors have shown clinical activity in the most common primary malignant brain tumor,

M.W. has received research grants from Abbvie, Acceleron, Actelion, Bayer, Merck, Sharp \& Dohme (MSD), Merck (EMD), Novocure, OGD2, Piqur, Roche, and Tragara and honoraria for lectures, advisory board participation, or consulting from Abbvie, BMS, Celldex, MSD, EMD, Novocure, Orbus, Pfizer, Progenics, Roche, Teva, and Tocagen.

${ }^{1}$ E.S. and D.J.P. contributed equally to this work.

https://doi.org/10.1124/jpet.117.246249.

S This article has supplemental material available at jpet.aspetjournals.org. glioblastoma, notably determined by responses on neuroimaging and prolongation of progression-free survival, no VEGF antagonist has been demonstrated to improve overall survival in any clinical setting in glioblastoma (Batchelor et al., 2014). Intratumoral and intertumoral heterogeneity, lack of evidence-based, rational combination therapies to overcome primary or acquired drug resistance, and poor tumor penetration leading to insufficient intratumoral drug concentrations are considered, among others, responsible for the failure of bevacizumab to improve overall survival in glioblastoma.

The penetration of an antibody into a tumor is a complex process that depends on a balance between molecular size and diffusion, affinity to the target, pharmacokinetics, tumor interstitial fluid pressure, dosing, and other factors (Beckman et al., 2007). It has long been known that the small size (Fig. 1, A and B) of single-chain antibody fragments (scFvs) allows them to penetrate faster, deeper, and more evenly into tumors than traditional IgG antibodies, which is also coupled with a faster blood clearance (Yokota et al., 1992). This increased and more homogeneous exposure of the tumor to antibody could offer a substantial advantage in the treatment of solid tumors; however, scFvs typically suffer from poor stability and have therefore not been developed as therapeutics to the same extent as IgG antibodies.

ABBREVIATIONS: CDR, complementarity-determining regions; hCMEC, human cerebral microvascular endothelial cells; SE-HPLC, size exclusion high-performance liquid chromatography; KinExA, kinetic exclusion assay; LDAO, N,N-dimethyldodecylamine N-oxide; PBS, phosphate-buffered saline; scFv, single-chain antibody fragment; VEGF-R, vascular endothelial growth factor-receptor. 


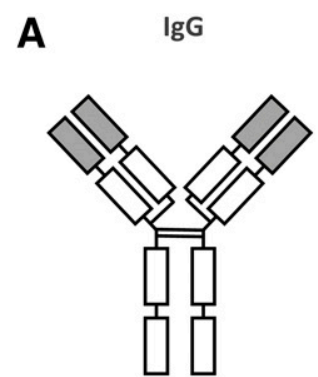

D

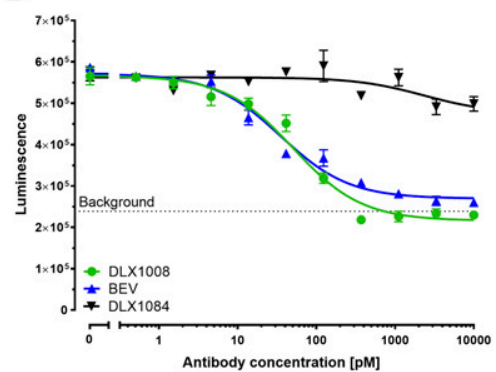

B

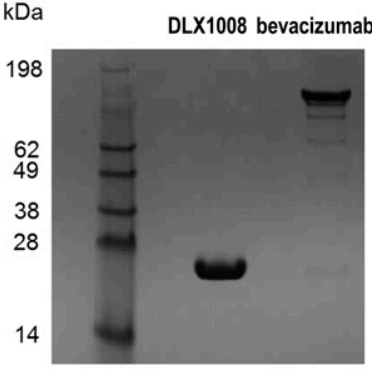

C

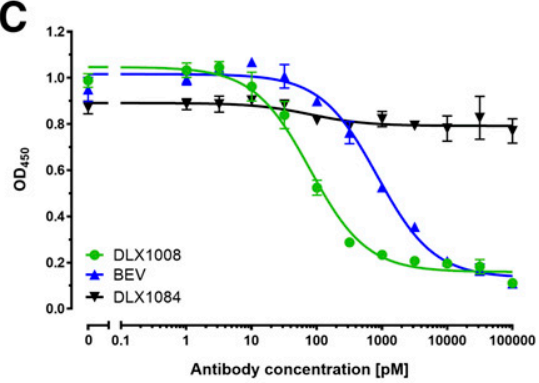

E

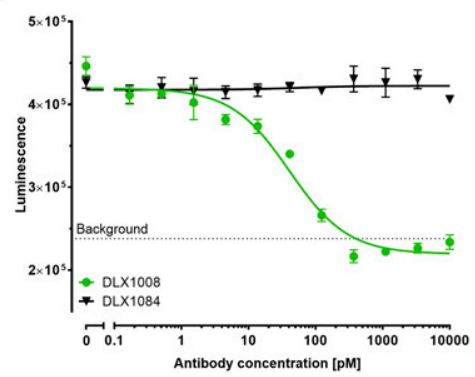

Fig. 1. Pharmacologic properties of DLX1008. (A) Structure of an IgG antibody and an scFv antibody fragment. Left: IgG antibodies, such as bevacizumab, typically have a molecular weight of around $150 \mathrm{kDa}$. The constant domains (white) provide stability and mediate effector functions, and the variable domains (gray) bind to antigens. Two antigen binding sites are present in each molecule. Right: ScFvs are the one of the smallest antibody fragments capable of target binding and typically have a molecular weight of around $25 \mathrm{kDa}$. They consist only of the variable domains and contain one binding site. (B) Gel electrophoresis comparing DLX1008 and bevacizumab. Ten micrograms of each protein was loaded. (C) DLX1008-, control scFv DLX1084-, or bevacizumab-mediated inhibition of VEGF-A 165 binding to VEGFR1 was assessed by enzyme-linked immunosorbent assay. (D and E) DLX1008-, control scFv DLX1084-, or bevacizumab-mediated inhibition of human VEGF-A 165 -stimulated (D) or DLX1008- or control scFv DLX1084-mediated inhibition of rat VEGF-A 164 -stimulated (E) VEGFR2 dimerization was measured in human embryonic kidney cells. Error bars represent S.E.M. of triplicate measurements (or duplicates for control scFv DLX1084 in the human receptor dimerization measurement).

Cell Medica's proprietary antibody discovery platform allows the development of exceptionally stable scFv antibodies. A number of highly stable human antibody frameworks have been identified through a process of stability selection using yeast cells (Auf der Maur et al., 2001). In this selection process, only those $\mathrm{scFv}$ frameworks that fold properly in the highly reducing intracellular yeast environment can express a reporter gene. These human antibody frameworks are not specific for any particular antigen, but can be used as scaffolds for the grafting of the binding regions (CDRs) from target specific antibodies. Target-specific CDRs can be obtained from numerous sources, including antibodies isolated from display libraries, animal immunization, or existing therapeutic antibodies, but one framework, known as the RABTOR framework, is particularly suitable for the engraftment of rabbit CDRs, which can be simply inserted into the relevant part of the framework (Borras et al., 2010). By this process, exceptionally stable, high-affinity humanized $\mathrm{scFvs}$ can be rapidly identified against a wide range of targets. Because of their high stability, these scFvs can be used as therapeutics in the $\mathrm{scFv}$ format. Alternatively, they can be combined to generate other antibody fragments or multispecific constructs, conjugated to drugs or radiolabels, or used as targeting agents in chimeric antigen receptor constructs for immune cell therapy.

DLX1008 is a VEGF-binding scFv, which binds with equal affinity to human and mouse VEGF (Dreier et al., 2014). It was isolated by rabbit immunization and humanized by grafting the rabbit CDR into a highly stable, proprietary human antibody framework. The same molecule, named brolucizumab, RTH258, or ESBA1008, is being developed by Novartis for use in ophthalmology (Holz et al., 2016) and has successfully completed phase 3 trials in age-related macular degeneration. In this article, we characterize the in vitro and in vivo characteristics of DLX1008.

\section{Materials and Methods}

Reagents. Recombinant human VEGF-A (carrier free), rat VEGF$\mathrm{A}_{164}$ (carrier free), and human VEGFR1-Fc (carrier free) were obtained from R\&D Systems (Abingdon, UK). VEGF-A 165 was obtained from Peprotech (London, UK). Cediranib (AZD2171) was purchased from BioVision (San Francisco, CA). Bevacizumab was obtained from Roche (Basel, Switzerland).

Cell Culture. Human cerebral microvascular endothelial cells (hCMEC/D3) were provided by P. C. Couraud (Paris, France). Human glioblastoma-derived endothelial cells (GMEC, ZHE-483-2) were isolated and cultured as described (Krishnan et al., 2015) using Lonza EBM-2 medium (CC-3156; Lonza, Walkersville, MD) with supplements (EGM-2-CC4176). U87MG glioma cells were obtained from the American Type Culture Collection (Manassas, VA) and have been extensively characterized in our laboratory (Weller et al., 1998). The cells are regularly authenticated at the Leibniz Institute DSMZGerman Collection of Microorganisms and Cell Cultures (Braunschweig, Germany) by short tandem repeat analysis. Human embryonic kidney cells engineered to coexpress VEGFR2 fused to ProLink or Enzyme Acceptor were obtained from DiscoverX (Fremont, CA).

Production of scFvs. BL21(DE3) Escherichia coli cells transformed with plasmids encoding scFvs were grown in LB medium and induced with isopropyl $\beta$-D-1-thiogalactopyranoside. After harvesting and resuspension of pellets in $10 \mathrm{mM}$ Tris buffer (TB), $150 \mathrm{mM} \mathrm{NaCl}$, $\mathrm{pH} 7.3$, cells were disrupted by the addition of lysozyme and 
sonification. Inclusion bodies were prepared by the addition of $\mathrm{N}, \mathrm{N}$-dimethyldodecylamine $\mathrm{N}$-oxide (LDAO) followed by centrifugation. Inclusion bodies were resuspended in TB with LDAO using a douncer, and the centrifugation/resuspension process was repeated three times with LDAO and twice without LDAO. The pellet was resuspended in $6 \mathrm{M}$ guanidine $\mathrm{HCl}, 100 \mathrm{mM}$ Tris, $1 \mathrm{mM}$ EDTA buffer, $\mathrm{pH}$ 8.5. Dithiothreitol $(20 \mathrm{mM})$ was added, and rapid dilution refolding was carried out by incubation overnight with $3 \mathrm{M}$ urea, $100 \mathrm{mM}$ Tris, $3 \mathrm{mM}$ cystine, $1 \mathrm{mM}$ cysteine, $\mathrm{pH}$ 8.5. DLX1084, an scFv of irrelevant specificity, was expressed and refolded similarly and served as a negative control. DLX1008 was concentrated, and buffer was exchanged to $50 \mathrm{mM}$ Tris, $\mathrm{pH} 8.5$, using tangential flow filtration. Purification was done by ion-exchange chromatography with a $\mathrm{Q}$ Sepharose FF (XK 26/20) column, followed by size exclusion chromatography using a Superdex 75 26/60 column. Control scFv DLX1084 was concentrated, and buffer was exchanged to $20 \mathrm{mM}$ sodium phosphate, $50 \mathrm{mM} \mathrm{NaCl}$, $\mathrm{pH} 7.0$, using tangential flow filtration. The sample was incubated for 30 minutes at room temperature with $\left(\mathrm{NH}_{4}\right)_{2} \mathrm{SO}_{4}$; the insoluble fraction was removed by centrifugation, and the supernatant was purified by hydrophobic interaction chromatography using a phenyl Sepharose 6 FF column, followed by size exclusion chromatography using a Superdex 75 26/60 column.

Gel Electrophoresis. Gel electrophoresis was carried out using a NuPAGE 12\% Bis-Tris gel (Thermo Fisher, Waltham, MA) and NuPAGE MES SDS Running Buffer (Thermo Fisher). Ten micrograms of DLX1008 and bevacizumab was loaded, alongside seeBlue Plus 2 prestained standard (Thermo Fisher). The gel was stained using $0.2 \%$ Coomassie Brilliant Blue, $45 \%$ ethanol, $40 \%$ acetic acid, and destained using $45 \%$ ethanol, $40 \%$ acetic acid.

Affinity Determination by Kinetic Exclusion Assay. The $K_{\mathrm{D}}$, on and off rates of DLX1008 and bevacizumab to VEGF-A ${ }_{165}$, were determined by kinetic exclusion assay (KinExA) with a KinExA 3200, including autosampler (Sapidyne Instruments, Boise, ID). This technique studies the interaction in solution, with a solid phase being used solely as a probe to detect the proportion of unbound antibody in the solution. Binding curves are constructed by titrating one binding partner while keeping the concentration of the other binding partner constant. Concentrations of each partner are chosen based on rangefinding experiments, to allow a full binding curve to be constructed. The use of binding curves at two different concentrations of constant binding partner allows the software to fit the binding parameters more precisely. All experiments were carried out in duplicate. All concentrations of VEGF-A 165 given were calculated using the molecular weight of the monomer. VEGF-A $\mathrm{A}_{165}$ was immobilized onto azlactone beads (Sapidyne Instruments) at a concentration of $10 \mu \mathrm{g} /$ $\mathrm{ml}$ in running buffer [phosphate-buffered saline (PBS), $\mathrm{pH} 7.4$, with $0.02 \% \mathrm{NaN}_{3}$ ). For the determination of DLX1008 affinity, two equilibrium binding curves were prepared in running buffer with $1 \mathrm{mg} / \mathrm{ml}$ bovine serum albumin. The first curve used an active concentration of $243 \mathrm{pM}$ DLX1008 and 15 2-fold dilutions of VEGF$\mathrm{A}_{165}$, starting with $2.5 \mathrm{nM}$, with an incubation time of 6 hours at $20^{\circ} \mathrm{C}$. The second curve used an active concentration of 24.3 pM DLX1008 and 152 -fold dilutions of VEGF-A $\mathrm{A}_{165}$, starting with $1 \mathrm{nM}$, with an incubation time of 19 hours at $20^{\circ} \mathrm{C}$. The amount of unbound antibody present in the mixtures was detected by injecting the samples onto the VEGF-A ${ }_{165}$-coated beads at a flow rate of $0.15 \mathrm{ml} / \mathrm{min}$. For both curves, captured DLX1008 was then detected by injecting $0.5 \mathrm{ml}$ of $250 \mathrm{ng} / \mathrm{ml}$ biotinylated Protein-L (GenScript, Piscataway, NJ), followed by $0.5 \mathrm{ml}$ of $250 \mathrm{ng} / \mathrm{ml}$ streptavidin DyLight 650 conjugate (Jackson ImmunoResearch, West Grove, PA), each at a flow rate of $0.25 \mathrm{ml} / \mathrm{min}$. The fluorescence signal, which is directly proportional to the concentration of free DLX1008 in the equilibrated samples, was converted to a voltage signal. This voltage signal was used to calculate the $K_{\mathrm{D}}$ value and activity of DLX1008 using the n-curve analysis equation of the KinExA Pro software version 4.1.9.

For the determination of bevacizumab affinity, two equilibrium binding curves were prepared in running buffer with $1 \mathrm{mg} / \mathrm{ml}$ bovine serum albumin and $0.5 \mathrm{mg} / \mathrm{ml}$ heparin. The first curve used an active concentration of $100 \mathrm{pM}$ bevacizumab and 142 -fold dilutions of VEGF$\mathrm{A}_{165}$, starting with $2 \mathrm{nM}$, with an incubation time of 13 hours at $20^{\circ} \mathrm{C}$. The second curve used an active concentration of $313 \mathrm{pM}$ bevacizumab and 152 -fold dilutions of VEGF-A $\mathrm{A}_{165}$, starting with $5 \mathrm{nM}$, with an incubation time of 6 hours at $20^{\circ} \mathrm{C}$. The amount of unbound antibody present in the mixtures was detected by injecting the samples onto the VEGF-A $\mathrm{A}_{165}$-coated beads at a flow rate of $0.15 \mathrm{ml} / \mathrm{min}$. For both curves, captured bevacizumab was detected by injecting $250 \mathrm{ng} / \mathrm{ml}$ goat antihuman IgG DyLight 650 conjugate (Pierce, Waltham, MA) at a flow rate of $0.25 \mathrm{ml} / \mathrm{min}$. The voltage signal was used to calculate the $K_{\mathrm{D}}$ value and activity of bevacizumab using the n-curve analysis equation of the KinExA Pro software version 4.1.9.

For determination of the DLX1008 on rate, the "kinetic inject" setup was used. Here, the amount of free $\mathrm{scFv}$ in samples with different VEGF- $\mathrm{A}_{165}$ is measured pre-equilibrium. An active concentration of 487 pM DLX1008, as well as 1 of 11 different VEGF-A ${ }_{165}$ concentrations (3-fold dilution series starting with $25 \mathrm{nM}$ ) was injected onto VEGF-A ${ }_{165}$-coated beads. Captured DLX1008 was detected as described to follow for affinity measurement. The $k_{\text {on }}$ values were determined using the kinetics inject equation in KinExA Pro software version 4.1.9. For determination of bevacizumab kinetics, the on rate was determined using the "kinetics direct" setup. An active concentration of $79 \mathrm{pM}$ bevacizumab and $125 \mathrm{pM}$ VEGF- $\mathrm{A}_{165}$ was mixed and injected onto VEGF-A $\mathrm{A}_{165}$ coated beads at different time points before equilibrium was reached to measure the percentage of unbound bevacizumab. Captured bevacizumab was detected as described herein for determination of $K_{\mathrm{D}}$. The $k_{\mathrm{on}}$ values were determined using the kinetics direct equation in KinExA Pro software version 4.1.9. All off rates were calculated multiplying by $K_{\mathrm{D}}$ and $k_{\text {on }}$.

Receptor Competition Assay. In vitro functionality of DLX1008 versus VEGFR1 was assessed by a receptor competition assay. VEGFR1-Fc was immobilized onto 96 -well plates overnight at $4^{\circ} \mathrm{C}$ at a concentration of $1 \mu \mathrm{g} / \mathrm{ml}$. Antibodies were preincubated with $131 \mathrm{pM}$ biotinylated human VEGF-A $\mathrm{A}_{165}$ for 1 hour and then added to the coated plates for 1 hour. Residual binding of VEGF-A ${ }_{165}$ to VEGFR1 was determined using a 30-minute incubation with streptavidin-HRP followed with tetramethylbenzidine (TMB) substrate.

Receptor Dimerization Assay. To explore whether the biologic activity of VEGF can be inhibited by DLX1008, a cellular human VEGFR2 dimerization receptor assay was used (PathHunter Bevacizumab Bioassay Kit; DiscoverX). Concentration response curves of VEGF-stimulated VEGFR2 dimerization were constructed using 0.01-1000 ng/ml human VEGF-A $A_{165}$ or rat VEGF-A ${ }_{164}$ and a 1:3 dilution series, with duplicate wells per concentration. DLX1008-, control scFv DLX1084-, or bevacizumab-mediated inhibition curves were constructed using $5 \mathrm{ng} / \mathrm{ml}$ human VEGF-A $\mathrm{A}_{165}$ or rat VEGF-A 164 and $0.2-3300$ or $0.1-10,000 \mathrm{pM}$ antibody, with triplicate wells per concentration. Background was calculated in the absence of VEGF and antibody.

Immunoblotting. Cells were seeded at subconfluent density in Dulbecco's modified Eagle's medium (DMEM) containing 10\% FCS, $1 \%$ glutamine and incubated overnight (12 hours). Cells were washed and serum-starved in the presence of equimolar concentrations (2683 nM) of DLX1008 or control scFv DLX1084 for 8 hours. A high concentration of VEGF is generally needed for VEGFR stimulation in

\section{TABLE 1}

Storage stability of DLX $1008^{a}$

\begin{tabular}{lcccc}
\hline \multicolumn{1}{c}{ Months of storage } & 0 & 1 & 2 & 12 \\
\hline Storage temperature & \multicolumn{4}{c}{ Monomer content (\%) } \\
$-20^{\circ} \mathrm{C}$ & 96 & 94 & 95 & 94 \\
$4^{\circ} \mathrm{C}$ & 96 & 94 & 95 & 94 \\
$22^{\circ} \mathrm{C}$ & 96 & 94 & 94 & n.d. \\
$37^{\circ} \mathrm{C}$ & 96 & 87 & 75 & n.d. \\
\hline
\end{tabular}

n.d., not determined.

${ }^{a} \mathrm{DLX} 1008$ was stored at $10 \mathrm{mg} / \mathrm{ml}$ in PBS, pH7.2, at different temperatures for different time points. Monomer content was assessed by SE-HPLC. 
TABLE 2

Freeze-thaw stability of DLX1008 ${ }^{a}$

\begin{tabular}{ccccc}
\hline Freeze-thaw cycles & 0 & 1 & 5 & 10 \\
\hline Monomer content $(\%)$ & 98 & 97 & 98 & 97 \\
\hline
\end{tabular}

${ }^{a} \mathrm{DLX} 1008$, formulated at $10 \mathrm{mg} / \mathrm{ml}$ in $\mathrm{PBS}, \mathrm{pH} 7.2$, was subjected to repeated freeze-thaw cycles. Monomer content was assessed by SE-HPLC.

glioma cells (Szabo et al., 2016). Therefore, a large amount of antibody is needed for neutralization, and the highest feasible concentration was used. VEGF112 (18 nM, calculated using the molecular weight of the dimer) was mixed and preincubated with $\mathrm{scFv}$ for 1 hour at room temperature in Eppendorf tubes before adding either one of these mixtures at $37^{\circ} \mathrm{C}$ for 15 minutes to the cells. The cells were lysed in icecold radio-immunoprecipitation assay buffer (Millipore, Temecula, CA), supplemented with protease and phosphatase inhibitors. Protein samples were loaded in equal concentrations $(30 \mu \mathrm{g})$ under reducing conditions. Quantification of protein band intensities was performed using Image $J$ (version 1.32j) software (National Institutes of Health, http://rsb.info.nih. gov/ij/). Human VEGFR1 was detected with goat anti-VEGFR1 (1:500) (Millipore, San Diego, CA), activated VEGFR1 with rabbit anti-phosphoVEGFR1 (Tyr1213) (1:5000) (Millipore). VEGFR2 was detected with rabbit anti-phospho-VEGFR2 (Tyr1175) and rabbit anti-total VEGFR2 (Cell Signaling Technology, Denvers, MA).
Stability. The stability of DLX1008 formulated in PBS, pH 7.2, was investigated after storage at different time points at $10 \mathrm{mg} / \mathrm{ml}$ at $4^{\circ} \mathrm{C}, 22^{\circ} \mathrm{C}, 37^{\circ} \mathrm{C}$, and $-20^{\circ} \mathrm{C}$. The samples were analyzed by size exclusion-high-performance liquid chromatography (SE-HPLC) to determine the levels (\%) of monomers, dimers, and high-molecularweight oligomers in relation to the total peak area: a TOSOH TSKgel G2000 SWXL column (Sigma, St Louis, MO) was used. Samples were centrifuged for 10 minutes at $16,100 \mathrm{~g}$, and the pellet was discarded; $5 \mu \mathrm{l}$ of supernatants was loaded onto the column. PBS, $\mathrm{pH} 7.2$, was used as mobile phase.

To assess the stability of DLX1008 during freeze/thaw cycles, it was formulated in PBS pH 7.2 at $10 \mathrm{mg} / \mathrm{ml}$. The vials were submerged into liquid nitrogen for 1 minute, then incubated in a water bath at room temperature for 5 minutes; three, 5,7 , or 10 freeze/thaw cycles were performed. Samples were centrifuged for 10 minutes at $16,100 \mathrm{~g}$, and the pellet was discarded. Supernatants were analyzed by SE-HPLC.

Tube Formation Assay. hCMECs were cultured in Corning BioCoat collagen type 1 cell culture plates (Corning, NY) in full EBM-2 medium overnight, then DLX1008, control scFv DLX1084, or bevacizumab were added to the medium. Since bevacizumab contains two binding sites and scFvs contain only one, the use of a 2 -fold lower bevacizumab concentration results in an equimolar binding site concentration. After 36 hours, the cells were transferred to drugcontaining growth factor-reduced matrigel (356230; BD Biosciences,
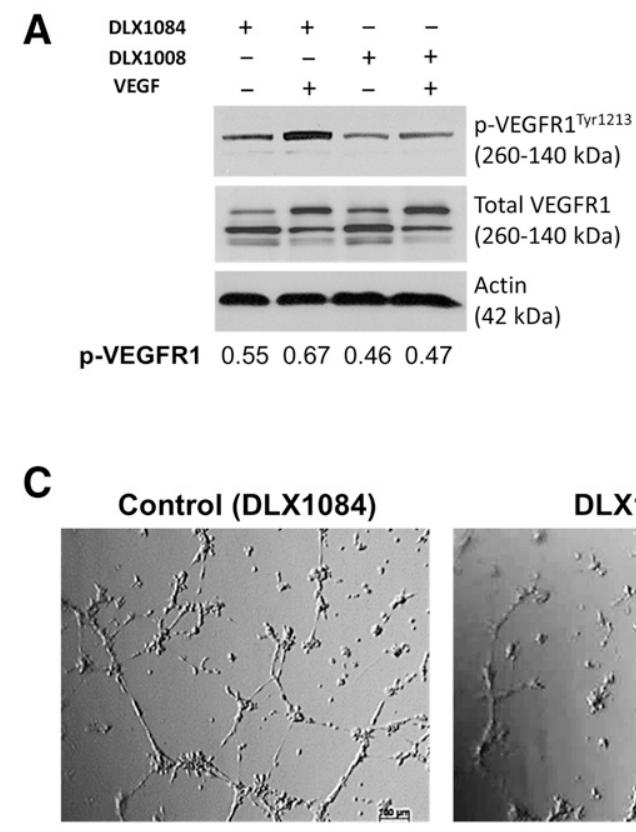

BEV

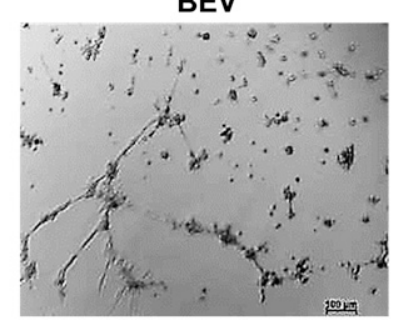

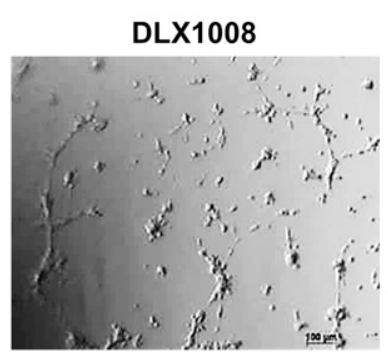

Cediranib

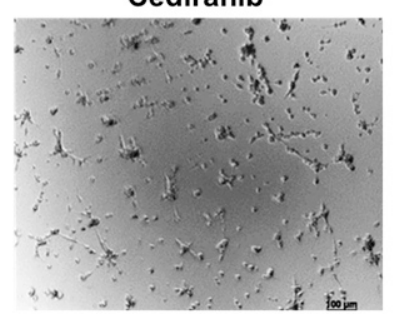

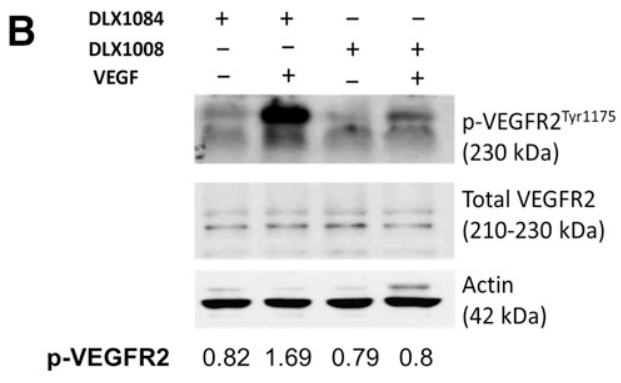

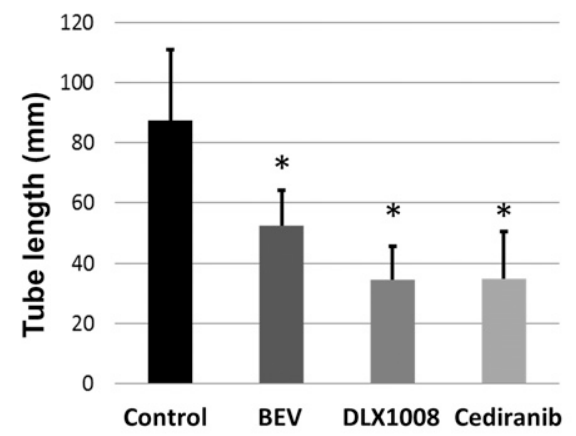

Fig. 2. DLX1008 inhibits VEGF signaling in human glioma and endothelial cells in vitro. (A and B) Receptor phosphorylation assays were carried out by immunoblot in human glioma cells (A) and GMEC (B). The effects of VEGF (18 nM, calculated using the molecular weight of the dimer, 15 minutes) stimulation on VEGFR1 $1^{\text {Tyr1213 }}$ (A) and VEGFR2 ${ }^{\text {Tyr1175 }}$ (B) were assessed when combined with control scFv DLX1084 or DLX1008 (2683 nM). Actin was used as a loading control. Signal intensities were normalized to actin, which showed no change on treatment. Quantification of band intensity is shown below the immunoblot panels. (C) Capillary tube formation assays were performed in growth factor-reduced Matrigel in the absence or presence of equimolar binding-site concentrations of antibodies, control scFv DLX1084 (2683 nM), DLX1008 (2683 nM), or bevacizumab (1342 nM) using hCMEC. Cediranib $(0.5 \mu \mathrm{M})$ was used as a positive control. Tube formation was quantified by assessing the length of the sprouts (millimeters). Significance $(* P<$ $0.05)$ is indicated. Data are expressed as mean value for biologic replicates \pm S.D. The statistical analyses were performed by Student's $t$ test. 
Franklin Lakes, NJ) in 96-well plates at 15,000 cells/130 $\mu$ l. Tube formation was assessed at 12 hours, and photographs were taken using a Carl Zeiss microscope (Axiovert 100; Göttingen, Germany). Tube length was measured in millimeters as an average of biologic replicates, each assayed in technical duplicate or triplicate using the Adobe Acrobat X Pro (Adobe, Ireland).

Animal Studies. Animal housing and experimental procedures were realized according to European and local regulations and the National Research Council Guide for the Care and Use of Laboratory Animals (Dijon, France, or Saarbrücken, Germany; Cantonal Veterinary Office Zurich, and Federal Food Safety and Veterinary Office). The effect of DLX1008 on subcutaneous tumor growth was examined in female RH nude rats (Hsd:RH-Foxn $1^{\text {rnu }}$; Harlan, Gannat, France) and female SWISS nude mice (Crl:NU(Ico)-Foxn $1^{\text {nu}}$ ) (Charles River, L'Arbresles, France). Twenty-four to 72 hours before cell injection, animals were irradiated with a $\gamma$-source (whole-body irradiation, 5 Gy (rats) or 2 Gy (mice), ${ }^{60} \mathrm{Co}$; INRA, Dijon, France) to improve the likelihood of tumor take. Tumors were induced subcutaneously by injecting $20 \times 10^{6}$ (rats) or $10 \times 10^{6}$ (mice) U87MG cells in $200 \mu \mathrm{l}$ of RPMI 1640 into the right flank. The day of tumor induction was considered day 0 . Treatment started when tumors reached a mean volume of $400-600 \mathrm{~mm}^{3}$, on day 19 (rats), or $100-200 \mathrm{~mm}^{3}$, on day 21 (mice). Fifty animals were randomized according to their individual tumor volume into six groups of 10 or 5 animals using Vivo manager software (Biosystemes, Couternon, France). Rats were treated once or twice daily with either 1 or $5 \mathrm{mg} / \mathrm{kg}$ DLX1008 by intraperitoneal injection. Mice were treated twice daily with $50 \mathrm{mg} / \mathrm{kg}$ DLX1008 by intraperitoneal injection. When dosing twice daily, the two administrations were spaced 6-8 hours apart. The length and width of the subcutaneous tumor were measured three times a week with calipers, and the volume of subcutaneous tumors was estimated by the formula [tumor volume $=\left([\text { width }]^{2} \times\right.$ length $\left.) / 2\right]$.

For intracranial tumor cell implantation, mice were anesthetized by an intraperitoneal injection of ketamine $70 \mathrm{mg} / \mathrm{kg}$ (ketamine 500 ; Centravet, Dinan, France) and xylazine $5 \mathrm{mg} / \mathrm{kg}$ (Rompun; Centravet) in $0.9 \% \mathrm{NaCl}$ solution at $10 \mathrm{ml} / \mathrm{kg}$ per injection. One hundred thousand $\left(10^{5}\right)$ U87MG cells suspended in $2 \mu \mathrm{l}$ of RPMI medium were stereotactically injected in the right frontal lobe of the mice. The cell suspension was injected at the rate of $0.5 \mu \mathrm{l} / \mathrm{min}$. The tumor cells were injected in the caudate nucleus of the right cerebral hemisphere. The day of tumor induction was considered day 0 . The mice were treated once or twice daily either with 5 or $50 \mathrm{mg} / \mathrm{kg}$ DLX1008 by intraperitoneal injection. When dosing twice daily, the two administrations were spaced 6-8 hours apart. Twice-weekly intraperitoneal injection of $5 \mathrm{mg} / \mathrm{kg}$ per day of bevacizumab was included as a reference in some experiments. The mean size of the intracranial tumors was estimated at the level of the largest tumor area of H\&Estained sections (Szabo et al., 2016).

Immunohistochemistry. In an experiment directly comparing tumor size and angiogenesis at the same time point, U87MG brain tumor samples were harvested from mice sacrificed when the first animal became symptomatic. Cryopreserved tumor sections were stained using the rat anti-CD31 (cluster of differentiation 31) (BD Pharmingen, San Diego, CA) and rabbit anti-phospho-VEGFR1

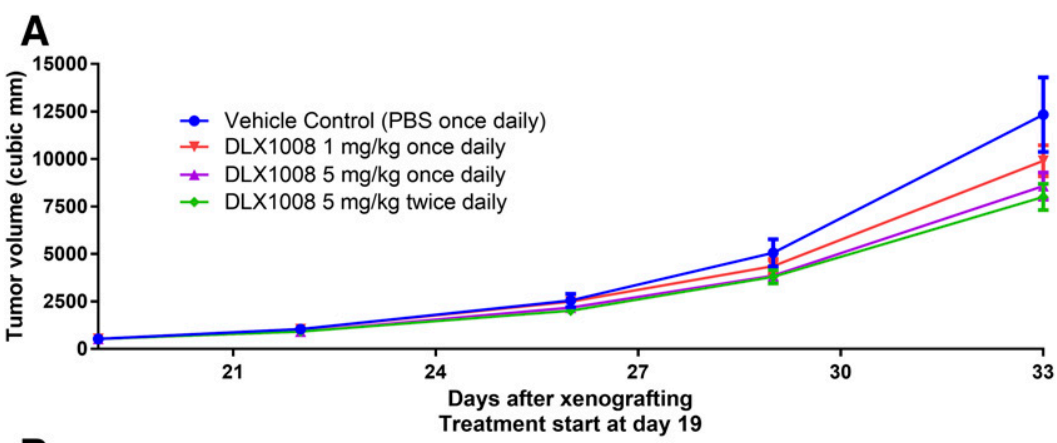

B

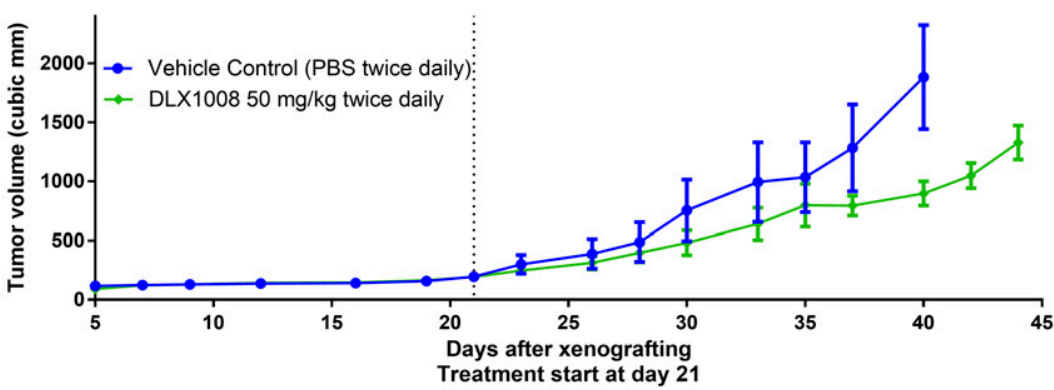

C

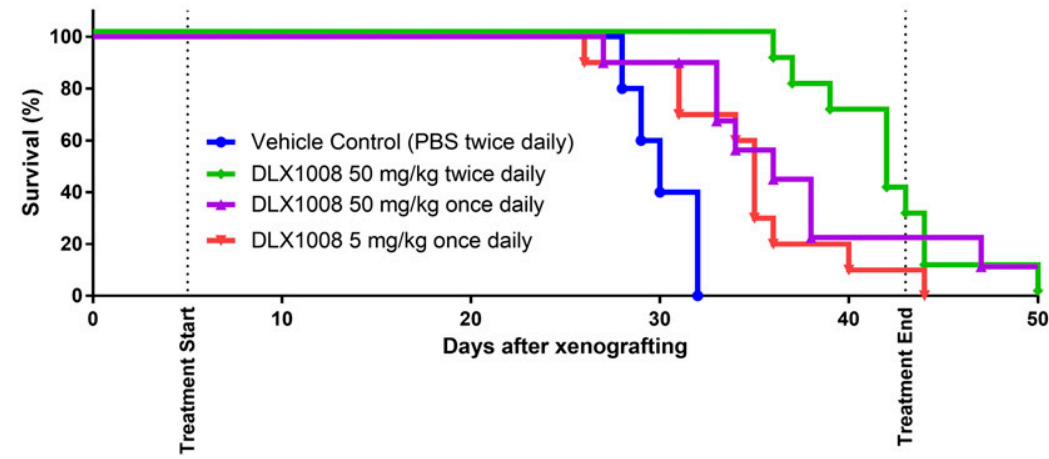

Fig. 3. DLX1008 delays in vivo tumor growth in U87MG glioma models. (A) For subcutaneous studies in rats, tumors were induced by injecting $20 \times 10^{6}$ U87MG cells in $200 \mu \mathrm{l}$ of RPMI 1640 into the right flank of $\mathrm{RH}$ nude rats. Treatment was started when tumors reached a mean volume of $400-600 \mathrm{~mm}^{3}$ and was maintained for $28 \mathrm{con}$ secutive days. Tumor volume was monitored by caliper measurements. Xenograft tumor volumes are shown as mean values \pm S.E.M. ( $n=10$ tumors except for the PBS control, $n=5$ ). (B) For subcutaneous studies in mice, tumors were induced by injecting U87MG cells in $200 \mu \mathrm{l}$ of RPMI 1640 into the right flank of Swiss nude mice. Treatment was started when tumors reached a mean volume of $100-200 \mathrm{~mm}^{3}$ and was maintained for 28 days. Tumor volume was monitored by caliper measurements. Xenograft tumor volumes are shown as mean values \pm S.E. M. $(n=10$ except for the PBS control, $n=5)$. (C) One hundred thousand $\left(10^{5}\right)$ U87MG cells suspended in $2 \mu \mathrm{l}$ of RPMI medium were stereotactically injected in the right frontal lobe of Swiss nude mice. The treatment was initiated on day 5 after tumor implantation and maintained until day 43. The survival curves show the percentage of living mice over time in all groups $(n=$ 10 except for the PBS control, $n=5$ ). 
(Tyr1213) (Millipore, Billerica, MA) primary antibodies, followed by the corresponding conjugated anti-host secondary antibodies: undiluted anti-rat Histofine Simple Stain Mouse MAX PO (Nichirei Biosciences, Tokyo, Japan) and 1:100 diluted horseradish peroxidase (HRP)-labeled anti-rabbit IgG secondary antibody (Santa Cruz Biotechnology, Santa Cruz, CA). To compare the immunoreactivities of each antigen between tumor specimens from the control and antiangiogenic treatment arms, quantification of the signal intensities of the stained area fractions was performed using Image $\mathrm{J}$ (version 1.32j) software by measuring the sum of the pixel values (integrated density) per field in the tumor region.

Statistical Analyses. Receptor competition and dimerization data are expressed as the mean \pm S.E.M. of three independent experiments in triplicate (DLX1008 and bevacizumab, also the control $\mathrm{scFv}$ DLX1084 for receptor competition) or duplicate (control scFv DLX1084 for receptor dimerization). Nonlinear regression was carried out using GraphPad Prism, version 6.05. A three-parameter log (inhibitor) versus response curve and weighting to $1 / \mathrm{Y}^{2}$ was used.

In vitro tube formation data were expressed as the mean and S.D. of two independent experiments in duplicate or triplicate. Statistical analysis of tube formation was performed by two-tailed Student's $t$ test and tumor volumes by analysis of variance. The log-rank (KaplanMeier) test was used to compare the survival curves. A $P$ value $<0.05$ was considered significant.

A

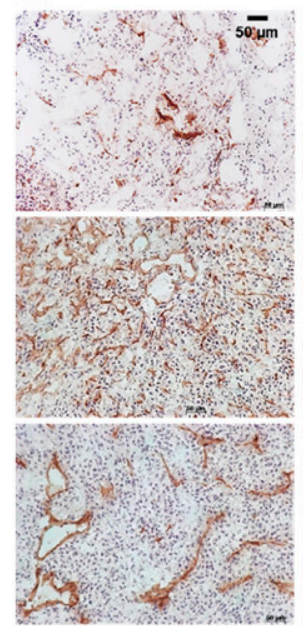

Control

\section{CD31}

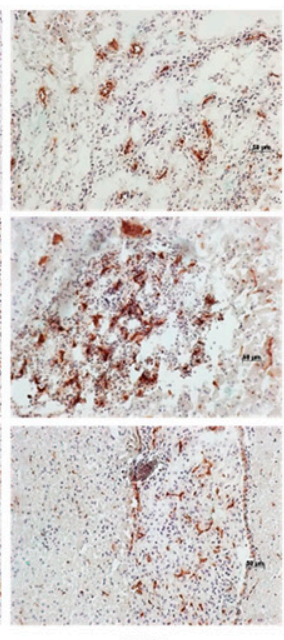

BEV

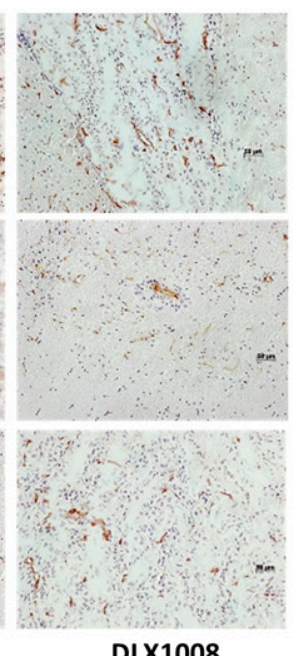

DLX1008

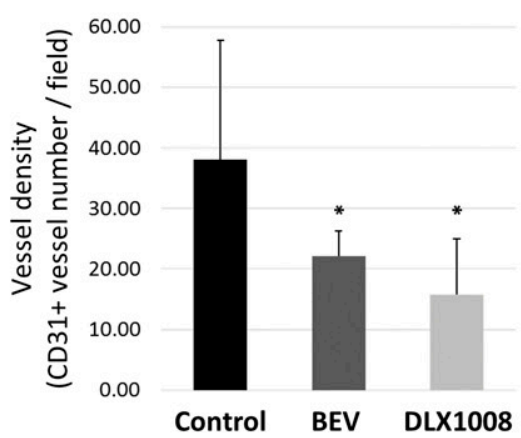

B

Control

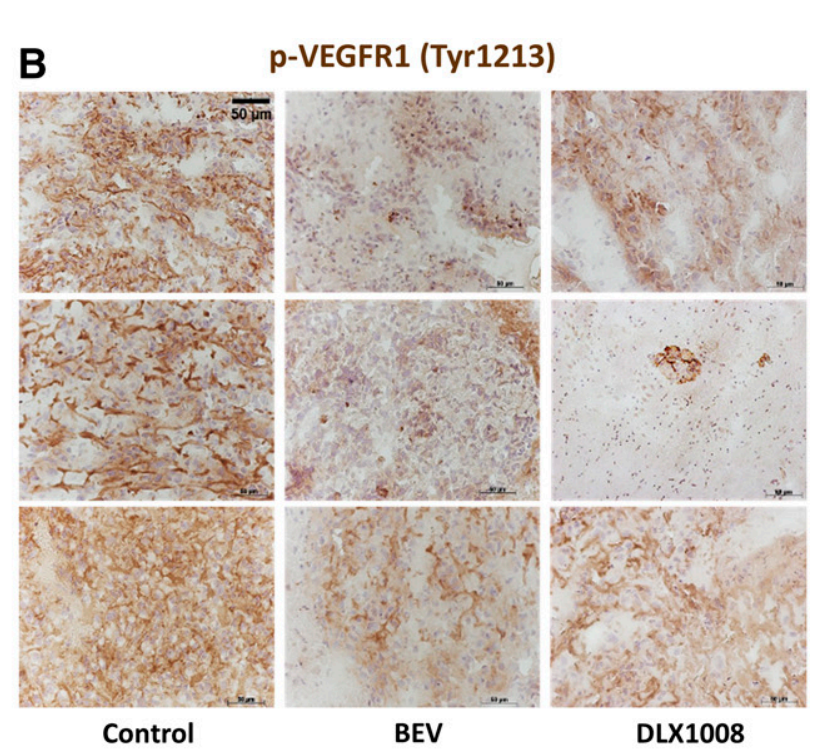

\section{Results}

DLX1008 is a Highly Stable, High-Affinity, Highly Potent Anti-VEGF Antibody. Two different processes affect the stability of scFvs. First, they can be prone to dimerization, often followed by oligomerization, further aggregation, and precipitation. Second, $\mathrm{scFv}$ degradation, leading to smaller fragments, can occur over time. The stability of DLX1008 formulated in PBS $\mathrm{pH} 7.2$ was investigated upon storage at different temperature conditions. Samples were analyzed by SE-HPLC to determine the levels (\%) of monomers, dimers, and high-molecular-weight oligomers in relation to the total peak area. Stability studies showed virtually no loss of monomer content and no protein precipitation, even after a year of storage at $4^{\circ} \mathrm{C}$. After 2 months of storage, the latest time point measured at room temperature, there was virtually no difference in the percentage of monomer content, measured by SE-HPLC, of samples stored at 4 or $22^{\circ} \mathrm{C}$, and the sample stored at $37^{\circ} \mathrm{C}$ still showed $75 \%$ monomer content (Table 1). The protein remained stable after 10 freeze-thaw cycles, with no protein precipitation or loss of monomer content (Table 2).

The affinity of DLX1008 for human VEGF-A 165 was determined by kinetic exclusion assay (KinExA). DLX1008 showed

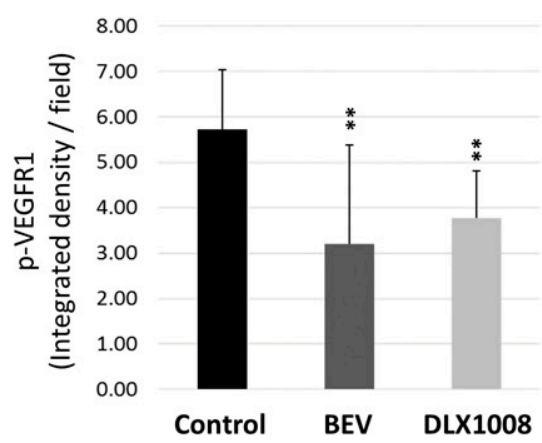

Fig. 4. DLX1008 inhibits tumor angiogenesis in the orthotopic U87MG mouse glioma model. (A and B) One hundred thousand U87MG cells were implanted into the right hemisphere of nude mice. Tumors were allowed to grow and develop a vascular network for 15 days after implantation, and mice with established tumors were then treated either twice daily with $50 \mathrm{mg} / \mathrm{kg}$ DLX1008 or control scFv DLX1084 or twice weekly with $5 \mathrm{mg} / \mathrm{kg}$ per day bevacizumab by intraperitoneal injection. U87MG tumor specimens were stained for CD31 and p-VEGFR1 (brown). Sections were counterstained with hematoxylin (blue). Representative immunohistochemistry staining for CD31 (A) and p-VEGFR1 ${ }^{\text {Tyr1213 }}$ (B) are shown for each mouse $(n=3$ per group, rows) in the control (left columns), bevacizumab-treated (middle columns), as well as DLX1008-treated (right columns) groups. Scale bars, $50 \mu \mathrm{m}$. Immunoreactivities of CD31 and p-VEGFR1 in the tumor region were assessed and expressed as the mean \pm S.D. $(n=3 ; * P<0.05$; ** $P<0.005, t$ test). 
exceptionally high affinity to human VEGF-A ${ }_{165}(1.05 \mathrm{pM}, 95 \%$ confidence interval $316 \mathrm{fM}-2.11 \mathrm{pM}$ ) (Supplemental Fig. 1, A-E), whereas bevacizumab showed an affinity of $32.4 \mathrm{pM}(95 \%$ confidence interval 13.3-59.1 pM) (Supplemental Fig. 1, F-J). DLX1008 showed an on rate $\left(k_{\text {on }}\right)$ of $5.04 \times 10^{6} \mathrm{M}^{-1} \mathrm{~s}^{-1}$ and an off rate $\left(\mathrm{k}_{\text {off }}\right)$ of $5.29 \times 10^{-6} \mathrm{~s}^{-1}$, whereas bevacizumab showed a $k_{\text {on }}$ of $4.13 \times 10^{5} \mathrm{M}^{-1} \mathrm{~s}^{-1}$ and a $k_{\text {off }}$ of $1.34 \times 10^{-5} \mathrm{~s}^{-1}$. Thus, the stronger binding of DLX1008 in comparison with bevacizumab is due to both a faster on rate and a slower off rate. In vitro functional activity of DLX1008 versus VEGFR1 was assessed by receptor competition assay (Fig. 1C). DLX1008 showed superior inhibition of VEGF ligand-receptor binding over bevacizumab (140 pM vs. $1200 \mathrm{pM}$ ). In vitro functionality through human VEGF stimulation of human VEGFR2 was assessed by receptor dimerization assay (Fig. 1D). DLX1008 was around half as potent as bevacizumab $(65.7 \pm 15.7 \mathrm{pM}$ vs. $31.9 \pm 4.3 \mathrm{pM})$; however, bevacizumab has two VEGF binding sites per molecule, whereas DLX1008 has only one. Accordingly, the potency per binding site is equal. DLX1008 was around 2-fold more potent for inhibition of human VEGFR2 dimerization stimulated by rat VEGF- $\mathrm{A}_{164}(35.5 \pm 4.5 \mathrm{pM})($ Fig. $1 \mathrm{E})$ compared with stimulation by human VEGF-A $\mathrm{A}_{165}$. Since the measured $\mathrm{IC}_{50}$ values in receptor competition and dimerization assays are lower than the concentration of VEGF- $\mathrm{A}_{165}$ used, the reported values are likely limited by the assay. Furthermore, DLX1008 neutralized VEGF-A A $_{12}$-induced phosphorylation of VEGFR1 in U87MG human glioma cells and of VEGFR2 in ZHE-483-2 GMEC as assessed by immunoblotting (Fig. 2, A and B).

In vitro antiangiogenic activity of DLX1008 was at least as high as that of bevacizumab in tube formation assays using HCMEC as target cells (Fig. 2C). Taken together, these data show that DLX1008 binds exceptionally tightly to VEGF-A and strongly inhibits interaction with its receptors.

DLX1008 Demonstrates Antitumor Activity in Human Glioma Xenograft Models In Vivo. Compared with vehicle control, DLX1008 retarded tumor growth as measured by tumor volumes in subcutaneous U87MG xenografts in both mice and rats. In nude rats, doses of $5 \mathrm{mg} / \mathrm{kg}$ once $(P=0.0091)$ and twice $(P=0.0030)$ daily retarded tumor growth (Fig. 3A).
Increasing the dose resulted in an increase in effect, and since the maximum effect did not appear to be reached, a second study was conducted using a higher dose, in mice instead of rats. In nude mice the $50 \mathrm{mg} / \mathrm{kg}$ twice daily dose showed efficacy $(P=0.0021$, Fig. 3B). In both the mouse and rat subcutaneous models, tumor growth is initially slow and starts to increase in the vehicle control groups around 19-22 days after tumor xenografting. DLX1008 slows tumor growth, with the gap between treatment and control groups increasing over time, but it does not result in tumor regression and thus most likely never a cure. In orthotopic U87MG xenografts in nude mice, treatment at all DLX1008 doses extended median survival $(P=$ $0.02711,0.00521$ and 0.00026 for $5 \mathrm{mg} / \mathrm{kg}$ once daily, $50 \mathrm{mg} / \mathrm{kg}$ once daily and $50 \mathrm{mg} / \mathrm{kg}$ twice daily, respectively) (Fig. 3C). DLX1008 was well tolerated at all doses in all studies. Similar to bevacizumab, DLX1008 therapy resulted in decreased CD31positive blood vessel density, as well as decreased p-VEGFR1 immunoreactivity in vascular structures and in glioma cells in the U87MG intracranial tumor (Fig. 4; Supplemental Fig. 2). Moreover, DLX1008 delayed intracranial tumor growth at least as efficiently as bevacizumab (Fig. 5). Taken together, these results show strong evidence for good tolerability and antitumor activity of DLX1008.

\section{Discussion}

ScFvs are one of the smallest antibody fragments $(26 \mathrm{kDa})$ that maintain the structure of the antigen binding site. This offers several advantages compared with standard monoclonal IgG antibodies $(150 \mathrm{kDa})$, such as improved tissue or tumor penetration and distribution (Beckman et al., 2007), and opens opportunities to explore alternative routes of administration. DLX1008 is a single-chain antibody fragment with exceptionally high affinity to VEGF ( $1 \mathrm{pM}$ as measured by Kinexa) and cross-reactivity to human, mouse and rat VEGF, in addition to other species.

Here we describe the preclinical evaluation of DLX1008 for its ability to induce antitumor responses against human glioblastoma models in vitro and in vivo.
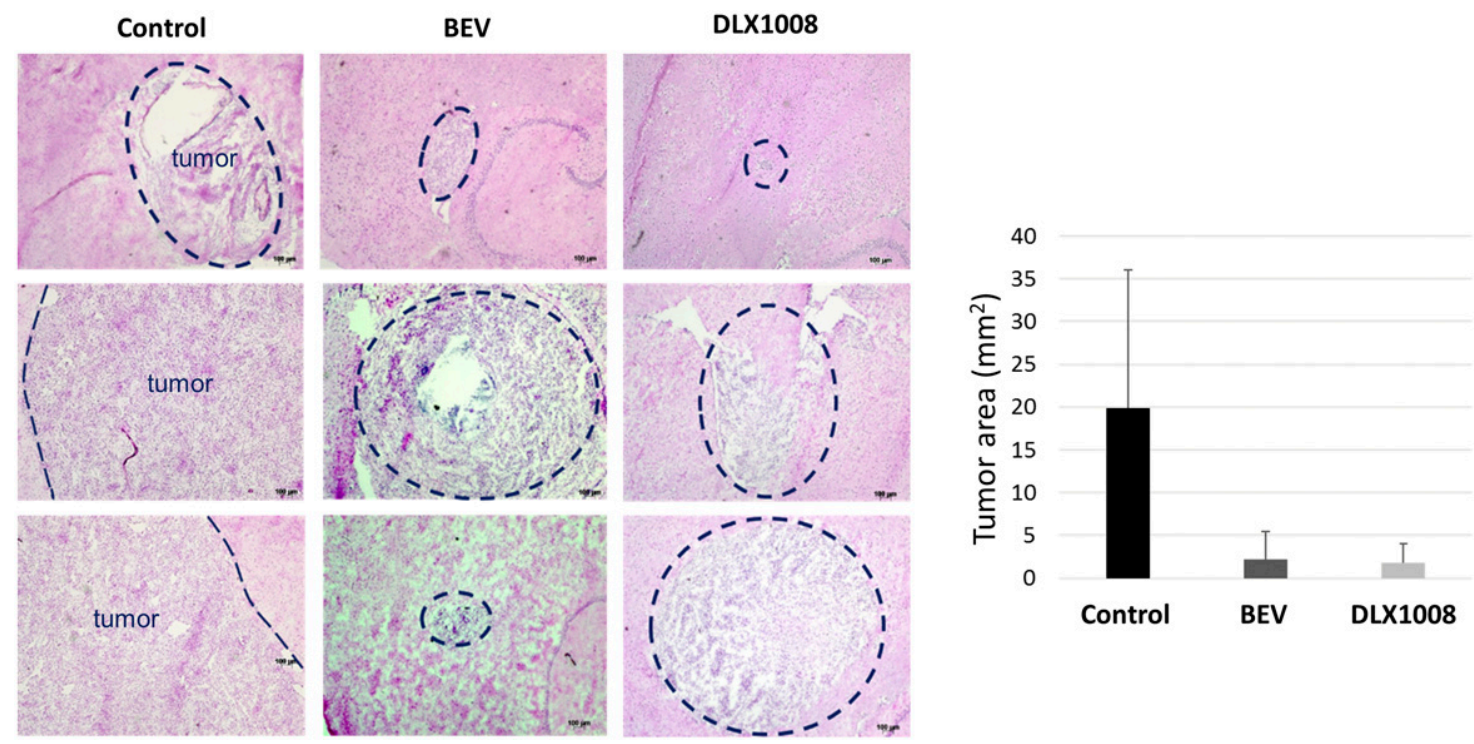

Fig. 5. Tumor growth inhibition by DLX1008 in U87MG mouse glioma model. Intracranial U87MG tumor sizes were determined based on H\&E-stained sections $(n=3 ; * P<0.05, t$ test) in the U87MG mouse glioma model generated and treated for 30 days as described above (Fig. 4). 
The affinity of bevacizumab measured in this study was 30-fold weaker than DLX1008 but is similar to that measured before using the same technique (Papadopoulos et al., 2012). Receptor competition and receptor dimerization assays demonstrated comparable or better potency of DLX1008 in the inhibition of VEGF ligand-receptor binding compared with bevacizumab when corrected for the number of binding sites (Fig. 1, C-E). Immunoblotting revealed that DLX1008 efficiently counteracted the binding and stimulation of VEGFR1 by VEGF-A 112 in U87MG human glioma cells as well as of VEGFR2 in GMEC (Fig. 2, A and B). Activity of DLX1008 in endothelial capillary tube formation assays in vitro was similar to that of bevacizumab (Fig. 2C). Moreover, DLX1008 significantly retarded tumor growth in subcutaneous U87MG tumor-bearing rats and mice, as well as prolonged survival in intracranial U87MG tumor-bearing mice (Fig. 3). This effect was associated with diminished levels of angiogenesis-associated markers (Fig. 4) and delayed tumor growth (Fig. 5). With regard to clinical applications, DLX1008 may offer improved efficacy compared with bevacizumab owing to increased affinity, better management of side effects resulting from faster pharmacokinetics and improved tumor penetration, given that DLX1008 is 6-fold smaller than bevacizumab. Another Cell Medica $\mathrm{scFv}$, the tumor necrosis factor-a targeting DLX105, which is also known as ESBA105, has been shown to penetrate effectively into the anterior and posterior segments of the eye when administered topically (Ottiger et al., 2009) and into the brain when administered intranasally (Hulmann-Cottier et al., 2014). Activity of DLX105 after topical administration in psoriasis patients has been confirmed by gene expression data in skin biopsies (Tsianakas et al., 2016). It would therefore be expected that DLX1008, which has similar features to ESBA105, will also show good tissue penetration.

The combination of antiangiogenic therapy with other treatment modalities has gained interest in glioblastoma (Seystahl et al., 2016); however, the combination of bevacizumab with lomustine failed to improve overall survival over lomustine alone in the European Organisation for Research and Treatment of Cancer 26101 trial (Wick et al., 2017).

Furthermore, extensive investigations on predictive biomarkers and mechanisms of resistance have led to a better understanding of sensitivity or resistance to bevacizumab therapy and provide new insight into putative novel approaches of combination therapies, for example, to overcome post-therapeutic metabolic reprogramming toward anaerobic metabolism or increased proinvasive tumor cell phenotype (Fack et al., 2015) or compensatory angiogenic pathways operating at cellular and molecular levels (Gacche, 2015). Nevertheless, enhanced drug delivery and tumor penetration are basic prerequisites of efficient therapy. Therefore, DLX1008 offers a promising perspective to develop alternative drug combinations with maximized anti-VEGF efficiency.

\section{Acknowledgments}

Nicole Dreier, Anna Howald, Marco Landi, and Julia Molitor provided technical support and scientific input.

\section{Authorship Contributions}

Participated in research design: Szabó, Phillips, Droste, Marti, Kretzschmar, Weller.
Conducted experiments: Szabó, Marti.

Contributed new reagents or analytic tools:

Performed data analysis: Szabó, Phillips, Droste, Marti, Kretzschmar, Weller.

Wrote or contributed to the writing of the manuscript: Phillips, Szabó, Kretzschmar, Shamshiev, Weller.

\section{References}

Auf der Maur A, Escher D, and Barberis A (2001) Antigen-independent selection of stable intracellular single-chain antibodies. FEBS Lett 508:407-412.

Batchelor TT, Reardon DA, de Groot JF, Wick W, and Weller M (2014) Antiangiogenic therapy for glioblastoma: current status and future prospects. Clin Cancer Res 20:5612-5619.

Beckman RA, Weiner LM, and Davis HM (2007) Antibody constructs in cancer therapy: protein engineering strategies to improve exposure in solid tumors. Cancer 109:170-179.

Borras L, Gunde T, Tietz J, Bauer U, Hulmann-Cottier V, Grimshaw JPA, and Urech DM (2010) Generic approach for the generation of stable humanized single-chain Fv fragments from rabbit monoclonal antibodies. J Biol Chem 285:9054-9066.

Dreier N, Phillips D, Shamshiev J, Steinwand M, Strassberger V, Grabulovski S, Howald A, Landi M, Marti A, Winnewisser C, et al. (2014) The PENTRA®Body platform: rapid discovery of highly potent femtomolar to low picomolar antibody fragments, in 6th Annual PEGS Europe, 3-7 November 2014, Lisbon, Portugal 10th Annual European Antibody Congress, 10-12 November 2014, Geneva, Switzerland, 25th Annual Antibody Engineering \& Therapeutics 7-11 December 2014, Huntington Beach, CA.

Fack F, Espedal H, Keunen O, Golebiewska A, Obad N, Harter PN, Mittelbronn M, Bähr O, Weyerbrock A, Stuhr L, et al. (2015) Bevacizumab treatment induces metabolic adaptation toward anaerobic metabolism in glioblastomas. Acta Neuropathol 129:115-131.

Gacche $\mathrm{RN}$ (2015) Compensatory angiogenesis and tumor refractoriness. Oncogenesis 4:e153.

Holz FG, Dugel PU, Weissgerber G, Hamilton R, Silva R, Bandello F, Larsen M, Weichselberger A, Wenzel A, Schmidt A, et al. (2016) Single-chain antibody fragment VEGF inhibitor RTH258 for neovascular age-related macular degeneration: a randomized controlled study. Ophthalmology 123:1080-1089.

Hulmann-Cottier V, Urech D, and Furrer E (2014) inventors, Esbatech An Alcon Biomedical Research Unit LLC, assignee. Methods and compositions for enhanced delivery of macromolecules WO/2010/003268, publication date 14th January 2010. Knizetova P, Ehrmann J, Hlobilkova A, Vancova I, Kalita O, Kolar Z, and Bartek J (2008) Autocrine regulation of glioblastoma cell cycle progression, viability and radioresistance through the VEGF-VEGFR2 (KDR) interplay. Cell Cycle 7: $2553-2561$.

Krishnan S, Szabo E, Burghardt I, Frei K, Tabatabai G, and Weller M (2015) Modulation of cerebral endothelial cell function by TGF- $\beta$ in glioblastoma: VEGFdependent angiogenesis versus endothelial mesenchymal transition. Oncotarget 6 : 22480-22495.

Lu KV, Chang JP, Parachoniak CA, Pandika MM, Aghi MK, Meyronet D, Isachenko N, Fouse SD, Phillips JJ, Cheresh DA, et al. (2012) VEGF inhibits tumor cell invasion and mesenchymal transition through a MET/VEGFR2 complex. Cancer Cell 22:21-35.

Ottiger M, Thiel MA, Feige U, Lichtlen P, and Urech DM (2009) Efficient intraocular penetration of topical anti-TNF-alpha single-chain antibody (ESBA105) to anterior and posterior segment without penetration enhancer. Invest Ophthalmol Vis Sci 50:779-786.

Papadopoulos N, Martin J, Ruan Q, Rafique A, Rosconi MP, Shi E, Pyles EA, Yancopoulos GD, Stahl N, and Wiegand SJ (2012) Binding and neutralization of vascular endothelial growth factor (VEGF) and related ligands by VEGF Trap, ranibizumab and bevacizumab. Angiogenesis 15:171-185.

Seystahl K, Wick W, and Weller M (2016) Therapeutic options in recurrent glioblastoma-an update. Crit Rev Oncol Hematol 99:389-408.

Szabo E, Schneider H, Seystahl K, Rushing EJ, Herting F, Weidner KM, and Weller M (2016) Autocrine VEGFR1 and VEGFR2 signaling promotes survival in human glioblastoma models in vitro and in vivo. Neuro-oncol 18:1242-1252.

Tsianakas A, Brunner PM, Ghoreschi K, Berger C, Loser K, Röcken M, Stingl G, Luger T, and Jung T (2016) The single-chain anti-TNF- $\alpha$ antibody DLX105 induces clinical and biomarker responses upon local administration in patients with chronic plaque-type psoriasis. Exp Dermatol 25:428-433.

von Baumgarten L, Brucker D, Tirniceru A, Kienast Y, Grau S, Burgold S, Herms J, and Winkler F (2011) Bevacizumab has differential and dose-dependent effects on glioma blood vessels and tumor cells. Clin Cancer Res 17:6192-6205.

Weller M, Rieger J, Grimmel C, Van Meir EG, De Tribolet N, Krajewski S, Reed JC, von Deimling A, and Dichgans J (1998) Predicting chemoresistance in human malignant glioma cells: the role of molecular genetic analyses. Int $J$ Cancer 79:640-644.

Wick W, Gorlia T, Bendszus M, Taphoorn M, Sahm F, Harting I, Brandes AA, Taal W, Domont J, Idbaih A, et al. (2017) Lomustine and bevacizumab in progressive glioblastoma. N Engl J Med 377:1954-1963.

Yokota T, Milenic DE, Whitlow M, and Schlom J (1992) Rapid tumor penetration of a singlechain Fv and comparison with other immunoglobulin forms. Cancer Res 52:3402-3408

Address correspondence to: Dr. Emese Szabó, Laboratory of Molecular Neuro-Oncology, Department of Neurology, University Hospital Zurich, Frauenklinikstrasse 26, 8091 Zurich, Switzerland. E-mail: emese.szabo@usz. ch; or Dr. Douglas J. Phillips, Cell Medica Switzerland AG, Wagistrasse 27, 8952 Schlieren, Switzerland. E-mail: infoag@cellmedica.com 